\title{
Impact of apatinib in combination with osimertinib on EGFR T790M-positive lung adenocarcinoma
}

\author{
Yang Liu ${ }^{1,2}$, Zhi-Cheng Xiong ${ }^{1,3}$, Xin Sun ${ }^{1,4}$, Li Sun ${ }^{1}$, Shu-Ling Zhang ${ }^{1}$, Jie-Tao Ma ${ }^{1}$, Cheng-Bo Han ${ }^{1}$ \\ ${ }^{1}$ Department of Oncology, ${ }^{2}$ Department of Anesthesiology, Shengjing Hospital of China Medical University, Shenyang 110022 , China; ${ }^{3}$ Department \\ of Radiotherapy, Xiamen Humanity Hospital of Fujian Medical University, Xiamen 361000, China; ${ }^{4}$ Department of Digestive Medical, Liaoning \\ Cancer Hospital, Shenyang 110022, China \\ Contributions: (I) Conception and design: CB Han; (II) Administrative support: CB Han, JT Ma; (III) Provision of study materials or patients: CB \\ Han; (IV) Collection and assembly of data: Y Liu, ZC Xiong, X Sun; (V) Data analysis and interpretation: Y Liu, ZC Xiong, L Sun, SL Zhang; (VI) \\ Manuscript writing: All authors; (VII) Final approval of manuscript: All authors. \\ Correspondence to: Cheng-Bo Han. Department of Oncology, Shengjing Hospital of China Medical University, No. 39 Huaxiang Road, Shenyang \\ 110022, China. Email: hanchengbo@sj-hospital.org.
}

Background: The purpose of this study was to investigate the anti-tumor activities and the mechanisms of the third-generation epidermal growth factor receptor (EGFR) tyrosine kinase inhibitor (TKI) osimertinib, combined with the anti-angiogenic target drug apatinib, in the treatment of lung adenocarcinoma. We investigated the effects of these drugs in vitro in PC9 (E19 del) and H1975 (E21 L858R/E20 T790M) cell lines, as well as in vivo in both mouse and human experiments.

Methods: PC9 and H1975 cells were cultured in 96-well plates and incubated with osimertinib (1-100 nmol/L), or apatinib (100-1,000 nmol/L), or a combination of the two agents, for 48 h. Cell viability was determined using a Cell Counting Kit-8. The protein expression of EGFR and its downstream signaling pathway members (AKT and ERK) was detected by western blot. For in vivo experiments, BALB/c nude mice were subcutaneously inoculated with H1975 cells in a xenograft model of adenocarcinoma. Mice bearing tumors were treated with osimertinib alone or in combination with apatinib, and tumor growth curves were obtained. Furthermore, we evaluated the efficacy and safety of combined osimertinib and apatinib therapy in three patients with EGFR T790M positive lung adenocarcinoma, who had been previously sensitized to osimertinib but developed an acquired resistance.

Results: In vitro experiments revealed that osimertinib combined with apatinib increased the growth inhibition of PC9 and H1975 cells, simultaneously reducing the protein expression of phosphorylated EGFR and its downstream signaling pathway members in H1975 cells, compared to osimertinib treatment alone. In vivo experiments revealed that the combination of osimertinib and apatinib decreased tumor volume in an H1975 cell xenograft model, compared to osimertinib monotherapy at different dosages. All three patients with T790M positive lung adenocarcinoma that progressed following osimertinib treatment responded to continuous osimertinib in combination with apatinib, with a progression-free survival (PFS) range of 5-7 months.

Conclusions: Apatinib can enhance the anti-tumor activity of osimertinib in the treatment of T790M positive lung adenocarcinoma. Further clinical studies are needed to confirm these results.

Keywords: Non-small cell lung cancer (NSCLC); epidermal growth factor receptor (EGFR); tyrosine kinase inhibitor (TKI); osimertinib; apatinib

Submitted Feb 24, 2019. Accepted for publication Aug 28, 2019.

doi: $10.21037 /$ tcr.2019.09.35

View this article at: http://dx.doi.org/10.21037/tcr.2019.09.35 


\section{Introduction}

Epidermal growth factor receptor (EGFR) tyrosine kinase inhibitors (TKIs) are the standard first-line therapy for patients with EGFR mutated advanced non-small cell lung cancer (NSCLC). First- or second-generation EGFR-TKIs can improve the objective response rate (ORR) by $60-80 \%$, as well as the median progression-free survival (PFS) by $9-13$ months (1-3). While most patients' cancer will eventually progress due to secondary resistance, approximately 50-60\% of cases are mediated by an acquired T790M mutation $(4,5)$. Osimertinib is a so-called third-generation EGFRTKI, targeting both EGFR sensitive mutations and the T790M mutation. A phase III study, AURA-3, indicated that osimertinib resulted in an ORR of $71 \%$ and a median PFS of 10.1 months for advanced NSCLC patients, who acquired the T790M mutation after resistance to first-line EGFRTKIs (6). The resistance mechanism of osimertinib is more complex compared to first- or second-generation EGFRTKIs. Currently, there are no effective targeted drugs to treat patients who have become resistant to osimertinib, with chemotherapy being the standard route of care.

Previous studies have shown that bevacizumab, an antivascular endothelial growth factor (VEGF) antibody, can enhance anti-tumor activity of chemotherapy and delay the onset of drug resistance (7). Preclinical studies have confirmed that EGFR-TKIs combined with bevacizumab can partially reverse the resistance to EGFR-TKIs $(8,9)$. Subsequently, a phase II clinical study has shown that EGFR-TKI combined with bevacizumab treatment results in a significantly prolonged PFS compared to EGFR-TKI alone (10). The reason for this may be that the EGFR and VEGF/VEGFR signaling pathways are tightly interconnected. EGFR-TKIs increase VEGF levels in tumors, which consequently negatively regulate the tumor vascular microenvironment. However, anti-angiogenic drugs can lower VEGF levels and angiogenesis, promote tumor vessel normalization, improve tumor oxygenation, decrease interstitial fluid pressure, and improve drug delivery to tumors, thereby inducing an increased sensitivity of the tumor cells to EGFR-TKIs (11).

Apatinib [AiTan ${ }^{\mathrm{TM}}$ (China); Rivoceranib ${ }^{\circledR}$ (global)] is a novel small molecule that is a selective VEGFR-2 TKI, which has been approved by the China Food and Drug Administration for the third-line treatment of advanced gastric cancer $(12,13)$. It can also effectively inhibit the activity of Ret, c-kit, c-src, and platelet-derived growth factor receptor $\beta$ (PDGFR- $\beta$ ), thus promoting anti-tumor activity (14). A phase II study indicated that apatinib had promising anti-tumor activity in advanced NSCLC, with lower and more manageable grade 3/4 toxicity events (15). A Phase III clinical study comparing the efficacy and safety of apatinib vs. placebo as a third-line treatment for EGFR mutation-negative advanced NSCLC is ongoing, and the results have yet to be reported.

Due to the multi-targeted anti-tumor effect and acceptable safety profile of apatinib, we aimed in the present study to investigate whether the combined therapy of a thirdgeneration EGFR-TKI, namely osimertinib and apatinib, could further improve the anti-tumor activity and delay the occurrence of acquired resistance for lung adenocarcinoma harboring the acquired EGFR T790M mutation, compared to single EGFR-TKI treatment alone.

\section{Methods}

\section{Cell culture and reagents}

The human NSCLC cell lines PC9 (exon 19 deletion) and H1975 (exon 21 L858R and exon 20 T790M) were obtained from the American Type Culture Collection. Cells were cultured in Dulbecco's modified Eagle's medium (DMEM) media (Biological Industries, Kibbutz Beit Haemek, Israel) or RPMI 1640 media (Biological Industries), supplemented with $10 \%$ fetal bovine serum (FBS) and $1 \%$ penicillinstreptomycin at $37{ }^{\circ} \mathrm{C}$ in $5 \%$ carbon dioxide. Osimertinib and apatinib were purchased from Selleck Chemicals (Shanghai, China).

\section{Cell growth assay}

PC9 and H1975 cell lines were seeded at a density of 2,000-5,000 cells per well in 96-well plates. After $24 \mathrm{~h}$ of incubation, cells were exposed to osimertinib (1-100 nmol/L), apatinib (100-1,000 nmol/L), or a combination of the two agents for $48 \mathrm{~h}$ in complete media. Cell viability was determined using a Cell Counting Kit8 (Biological Industries). The absorbance was measured at $450 \mathrm{~nm}$ based on the principle of water-soluble formazan dye development from tetrazolium salt. The dose administered was based on the results of the preliminary experiment and data from other articles. Data are shown as the mean \pm standard deviation (SD) of at least three independent experiments performed in triplicate. 


\section{Western blotting}

Cells (H1975) were seeded into 6-well plates at a concentration of $5 \times 10^{5}$ cells per well, and were incubated overnight. Then, osimertinib, apatinib or a combination of the two agents were added, and cells were incubated continuously for $48 \mathrm{~h}$. Cells were lysed in radio immunoprecipitation assay (RIPA) buffer containing phenylmethanesulfonyl fluoride (PMSF) with mild sonication. The total protein concentration was measured using a BCA Protein Assay Kit (Beyotime Biotechnology, Beijing, China). Equal amounts of protein $(40-50 \mathrm{mg}$ ) were subjected to sodium dodecyl sulfate-polyacrylamide gel electrophoresis (SDS-PAGE) using 10\% Bis-Tris precast gels (Beyotime Biotechnology), and transferred onto polyvinylidene difluoride (PVDF) membranes (Millipore, Hayward, CA, USA) using the semi-dry transfer system. The membranes were blocked in $5 \%$ bovine serum albumin-tris-buffered saline with Tween (BSA-TBST) or milk-TBST for $1 \mathrm{~h}$ at room temperature, and blotted with primary antibodies against total EGFR, phospho-EGFR, total AKT, phospho-AKT, total ERK, phospho-ERK, or GAPDH at $4{ }^{\circ} \mathrm{C}$ overnight. Membranes were then incubated with horseradish peroxidase (HRP)-conjugated goat antirabbit secondary antibodies or anti-mouse secondary antibodies for $2 \mathrm{~h}$ at room temperature, and visualized by electrochemiluminescence (ECL). The image was scanned and analyzed using Image J (National Institutes of Health, Bethesda, MA, USA), and protein levels were normalized to the level of GAPDH.

\section{Animals and tumor xenograft experiments}

Five to 6-week-old female BALB/c nude mice were purchased from Beijing Huafukang Biotechnology Co. (Beijing, China). Mice were provided with food and water, and housed under a $12 \mathrm{~h}$ light-dark cycle. Mice were allowed to acclimatize for 1 week before any handling. The experimental protocols were reviewed and approved by the China Medical University Animal Care and Use Committee (Shenyang, China). The study was conducted by the National Institutes of Health "Principles of Laboratory Animal Care" (NIH publication No. 85-23, revised 1985) guidelines for the care and use of laboratory animals.

H1975 cells were harvested and resuspended in a 4:1 mixture of serum-free RPMI 1640 and Matrigel. Cells $\left\{[5-10] \times 10^{6}\right\}$ were injected subcutaneously into the rear flank of mice using a 20 -gauge needle. After two weeks, when tumors had grown to $400-600 \mathrm{~mm}^{3}$, mice were randomized into three groups for dosing: a low dose of osimertinib $(\mathrm{n}=4,2.5 \mathrm{mg} / \mathrm{kg} /$ day, p.o. gavage; group A), a high dose of osimertinib ( $\mathrm{n}=4,5 \mathrm{mg} / \mathrm{kg} /$ day, p.o. gavage; group B) and a low dose of osimertinib plus apatinib $(n=4$, osimertinib $2.5 \mathrm{mg} / \mathrm{kg} /$ day, p.o. gavage; apatinib $2.5 \mathrm{mg} / \mathrm{kg}$, twice weekly, p.o. gavage; group C). The dose administered was based on the results of the preliminary experiment and data from other articles. The tumor volumes were measured using a digital caliper twice weekly, and were calculated using the following equation: volume $(\mathrm{V})\left(\mathrm{mm}^{3}\right)=$ length $\times$ width $^{2} \times \pi / 6$. Moribund animals were killed to reduce suffering. After 16 days of treatment, mice were euthanized.

\section{Patients}

The efficacy and safety of combined therapy of osimertinib and apatinib was evaluated in eligible patients with EGFR T790M positive lung adenocarcinoma. Eligible patients were previously sensitized to osimertinib, but developed acquired resistance. The enrolled patients were treated with continuous osimertinib $(80 \mathrm{mg} /$ day) plus apatinib $(500 \mathrm{mg} /$ day $)$ in the hospital, until the disease progressed according to the Response Evaluation Criteria in Solid Tumor Standard (version 1.1), or until unacceptable toxicities developed. All patients were followed up once every 2 months for a chest and abdominal CT scan. All patients provided written informed consent prior to participating in the study. This study was approved by the ethics committee of our hospital, and the study was conducted in accordance with the Declaration of Helsinki.

\section{Statistical analysis}

All data were analyzed using SPSS software, version 19.0 (SPSS, Chicago, IL, USA). Quantitative data are expressed as the mean \pm SD. A Student's $t$-test was used for comparisons between two groups. A P value less than 0.05 was considered statistically significant.

\section{Results}

\section{Inbibitory effect of osimertinib combined with apatinib on PC9 and H1975 cells in vitro}

The inhibition rate of PC9 and H1975 cells in each group is shown in Figure 1A,B,C,D. PC9 and H1975 lung adenocarcinoma cells were sensitized to osimertinib or 
A
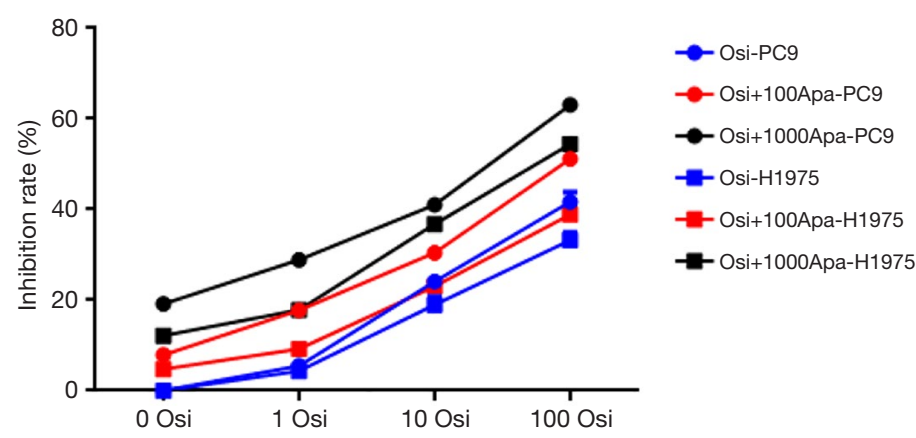

C

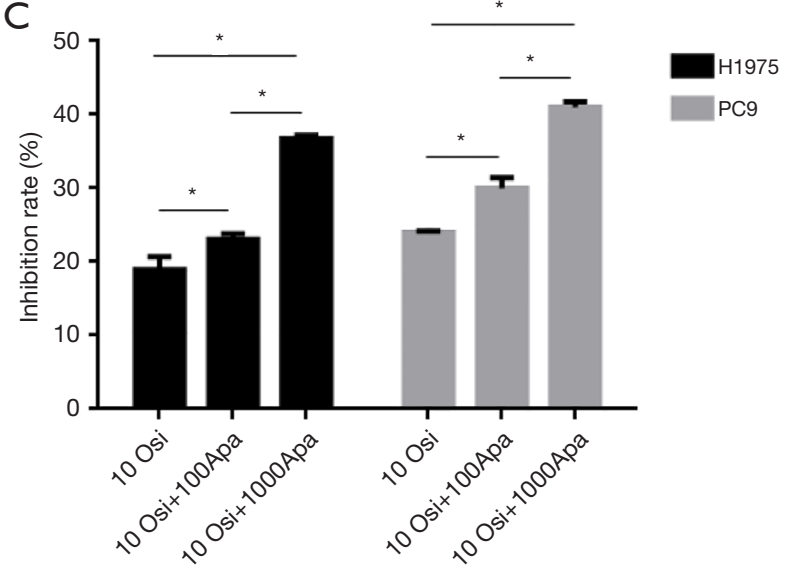

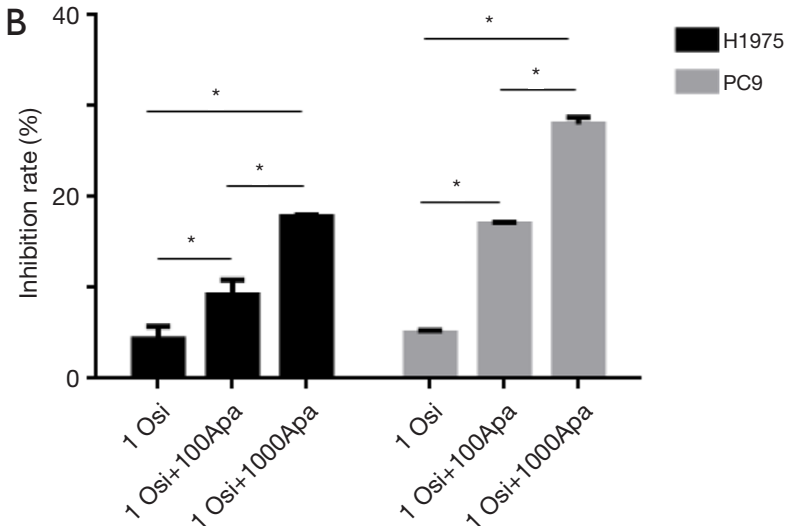
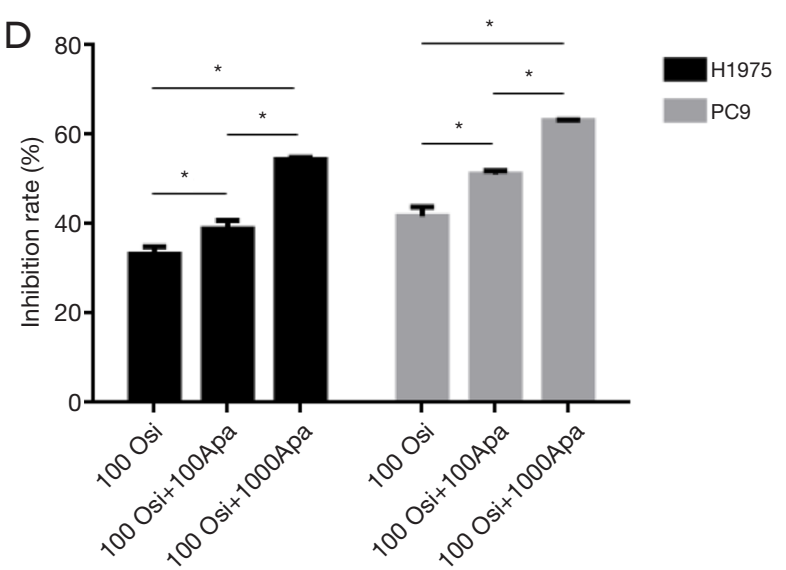

Figure 1 Inhibition rate of PC9 and H1975 cells after exposure to osimertinib or combined apatinib for 48 h. (A) Cure graph. (B,C,D) Column graph. *, $\mathrm{P}<0.05$. The units of osimertinib and apatinib are nmol/L. Osi, osimertinib, Apa, apatinib.

osimertinib combined with apatinib in a dose-dependent manner. The inhibitory rate of PC9 cells was significantly higher than that of H1975 cells, at all drug concentrations $(\mathrm{P}<0.01)$. Osimertinib combined with apatinib increased the tumor inhibition rate of PC9 and H1975 cell lines, compared to single osimertinib treatment at the same concentration $(\mathrm{P}<0.05)$. Under a fixed concentration of osimertinib, the tumor inhibition rate of PC9 and H1975 cells also increased to a certain extent with increased concentration of apatinib $(\mathrm{P}<0.05)$. Additionally, the tumor inhibition rates under low concentrations of osimertinib combined with a high concentration of apatinib $(1,000$ $\mathrm{nmol} / \mathrm{L}$ ) were similar to that of a high concentration of osimertinib alone $(\mathrm{P}>0.05)$.

\section{EGFR and its downstream signaling protein expression in H1975 cells}

Western blot results are shown in Figure 2. The protein expression of p-EGFR, p-AKT, and p-ERK gradually decreased with increased osimertinib concentration. Additionally, the expression levels of p-EGFR, p-AKT, and $\mathrm{p}$-ERK in the osimertinib combined with apatinib group were modestly decreased, compared to treatment with apatinib or osimertinib alone $(\mathrm{P}<0.05)$. Apatinib monotherapy resulted in negligible inhibition of protein expression. Total EGFR, total AKT, and total ERK protein expression was unchanged in each group.

\section{Anti-tumor activity of osimertinib combined with apatinib in xenograft models}

The tumor growth in each group of mice after two weeks of treatment are shown in Figure 3A,B,C. Osimertinib monotherapy or a combination of osimertinib and apatinib resulted in tumor inhibition and a reduced tumor volume of H1975 lung adenocarcinoma cell xenograft models, to differing levels. Specifically, tumor volume was more highly 


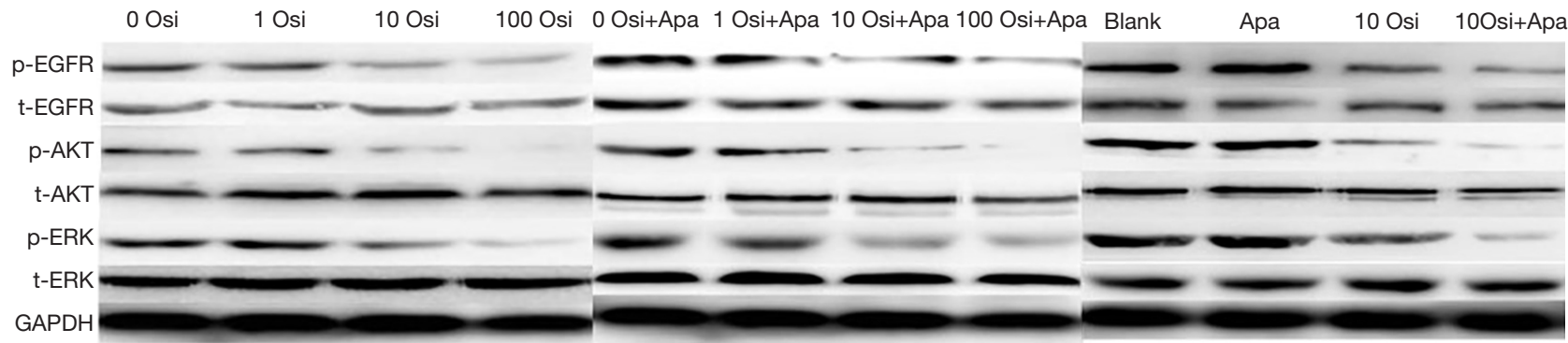

Figure 2 Effect of osimertinib and apatinib on EGFR and its downstream signaling pathway protein expression in H1975 cells. The units of osimertinib and apatinib are nmol/L. Osi, osimertinib, Apa, apatinib; EGFR, epidermal growth factor receptor.
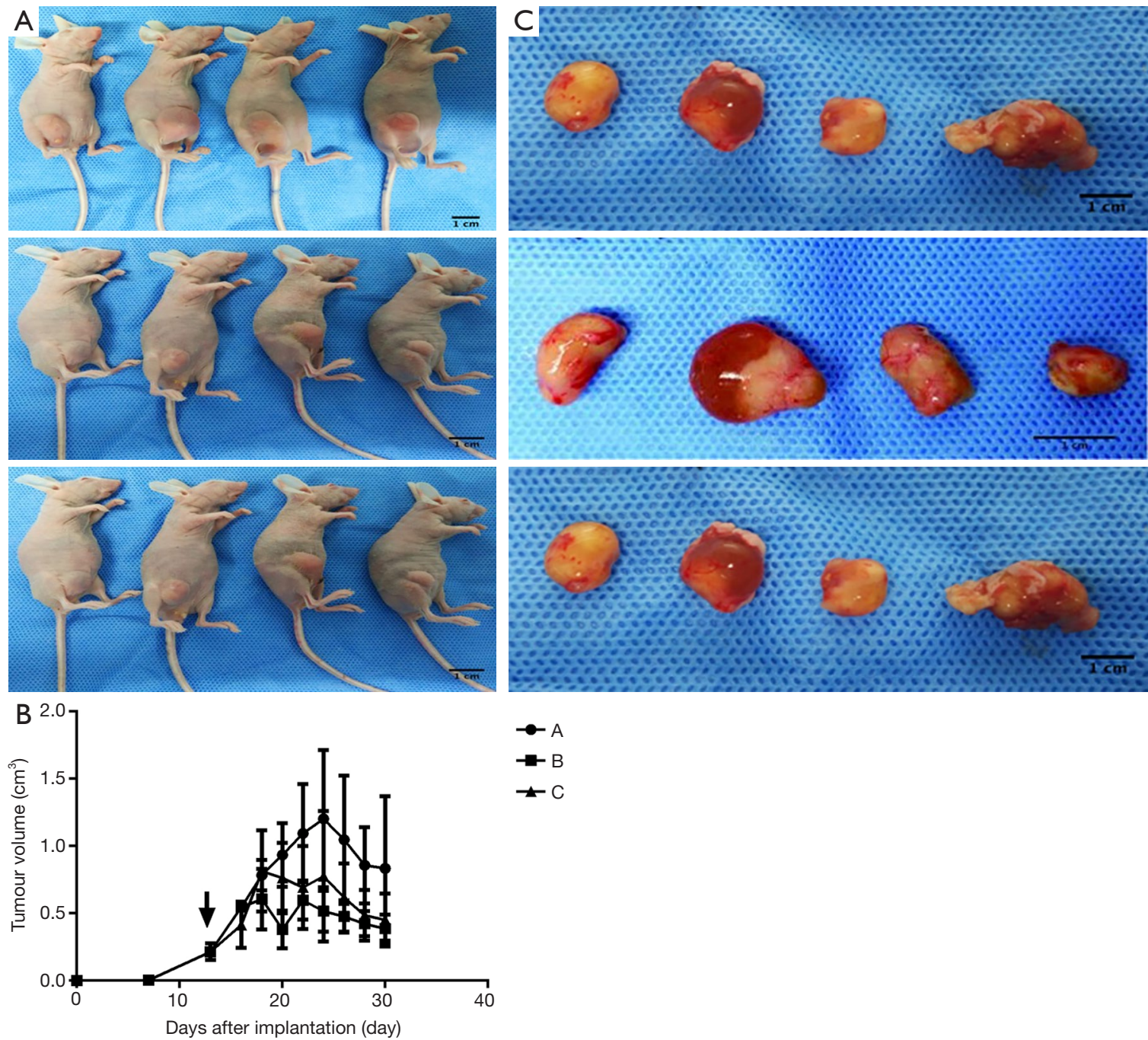

Figure 3 Results of osimertinib monotherapy vs. combination of osimertinib and apatinib in H1975 cell xenograft models. (A) Images of nude mice in each group. Mice were treated on day 14 with osimertinib alone at $2.5 \mathrm{mg} / \mathrm{kg} / \mathrm{day}$, A group; osimertinib alone at $2.5 \mathrm{mg} / \mathrm{kg} / \mathrm{day}$, B group; osimertinib $2.5 \mathrm{mg} / \mathrm{kg} / \mathrm{day}$ and apatinib $2.5 \mathrm{mg} / \mathrm{kg}$, twice weekly, C group. After 16 days of treatment, mice were euthanized. (B) Tumor volume growth curve. Each point represents the mean \pm standard deviation of tumor volumes from four mice in each group. The black arrow indicates that the mice were randomly intervened with drugs on the 14th day after inoculation. (C) Images of tumor samples in each group. The samples were taken on day 30 after implantation. 
Table 1 Characteristics of three advanced lung adenocarcinoma patients administered therapy

\begin{tabular}{|c|c|c|c|}
\hline Characteristics & Patient \#1 & Patient \#2 & Patient \#3 \\
\hline Sex & Male & Male & Male \\
\hline Stage & IV (T2N0M1) & IV (T4N2M1) & II (T2N1M0) \\
\hline EGFR mutation & Exon21 L858R & Exon19 del & Exon19 del \\
\hline \multicolumn{4}{|l|}{ Treatment [duration, months] } \\
\hline Adjuvant therapy & - & - & $\mathrm{DDP}+\mathrm{TAX}$ \\
\hline First-line & DDP + PEM + PRT & ERL [10] & $\mathrm{DDP}+\mathrm{PEM}$ \\
\hline Maintenance & PEM then ERL [45] & - & PEM then ERL [20] \\
\hline Fourth-line & Osi + Apa [5] & Osi [5] & Osi + Apa + PRT [9] \\
\hline Fifth-line & - & Osi + Apa [7] & Bev + DTX + Gem [1] \\
\hline Sixth-line & - & $\mathrm{S}-1+$ Oxa $[6+]$ & Osi [8+] \\
\hline $\mathrm{OS}_{1}$ (months) & 65 & $43+$ & $90+$ \\
\hline $\mathrm{OS}_{2}$ (months) & 6 & $13+$ & $13+$ \\
\hline Adverse effects & G3 oral mucositis, G3 fatigue & G2 fatigue & G2 oral mucositis, G3 fatigue \\
\hline
\end{tabular}

$\mathrm{OS}_{1}$ was defined as the time from the start of first line of treatment to the date of death by any cause; OS ${ }_{2}$ was defined as the time from the start of apatinib combined with osimertinib treatment to the date of death by any cause. DDP, cisplatin; PEM, pemetrexed; PRT, palliative radiotherapy; Osi, osimertinib; Apa, apatinib; ERL, erlotinib; AB-TAX, albumin-bound taxol; WBRT, whole-brain radiotherapy; TMZ, temozolomide; DTX, docetaxel; NDP, nedaplatin; Bev, bevacizumab; Gem, gemcitabine; Oxa, oxaliplatin; G2/3, grade 2/3.

reduced in the low-concentration osimertinib combined with apatinib group and high-concentration osimertinib monotherapy group, compared to the low-concentration osimertinib monotherapy group $(\mathrm{P}<0.05)$. In addition, the anti-tumor efficacy in the low-concentration osimertinib combined with apatinib group was equal to that of the highconcentration osimertinib monotherapy group $(\mathrm{P}=0.83)$.

\section{Apatinib in combination with osimertinib in osimertinib- resistant patients}

The characteristics, efficacy, and safety of three patients treated with osimertinib in combination with apatinib are shown in Table 1. All three patients who progressed with prior osimertinib treatment responded to continuous osimertinib plus additional apatinib, with an ORR of $100 \%$ and a PFS range of 5-7 months. The CT/MRI scans are shown in Figure 4A,B,C,D. The most common grade 3 adverse events were fatigue, oral mucositis, and anorexia.

\section{Discussion}

In clinical practice, osimertinib is commonly used for the treatment of patients who have acquired the EGFR T790M mutation in advanced NSCLC after secondary resistance to prior first- or second-generation EGFR-TKIs. Osimertinib remarkably improves median PFS compared to the first generation of EGFR-TKIs used as first-line treatments for EGFR sensitizing mutation-positive NSCLC (18.9 versus 10.2 months) (16). Nevertheless, patients who initially respond to osimertinib will inevitably develop resistance. Our study shows that osimertinib in combination with apatinib in the treatment of T790M mutation-positive lung adenocarcinoma can increase anti-tumor efficacy, compared to osimertinib monotherapy. It may even overcome the resistance to osimertinib in patients who initially progressed on osimertinib, resulting in an impressive PFS and manageable toxicity profile. Therefore, the effect of delaying and overcoming the occurrence of resistance to 

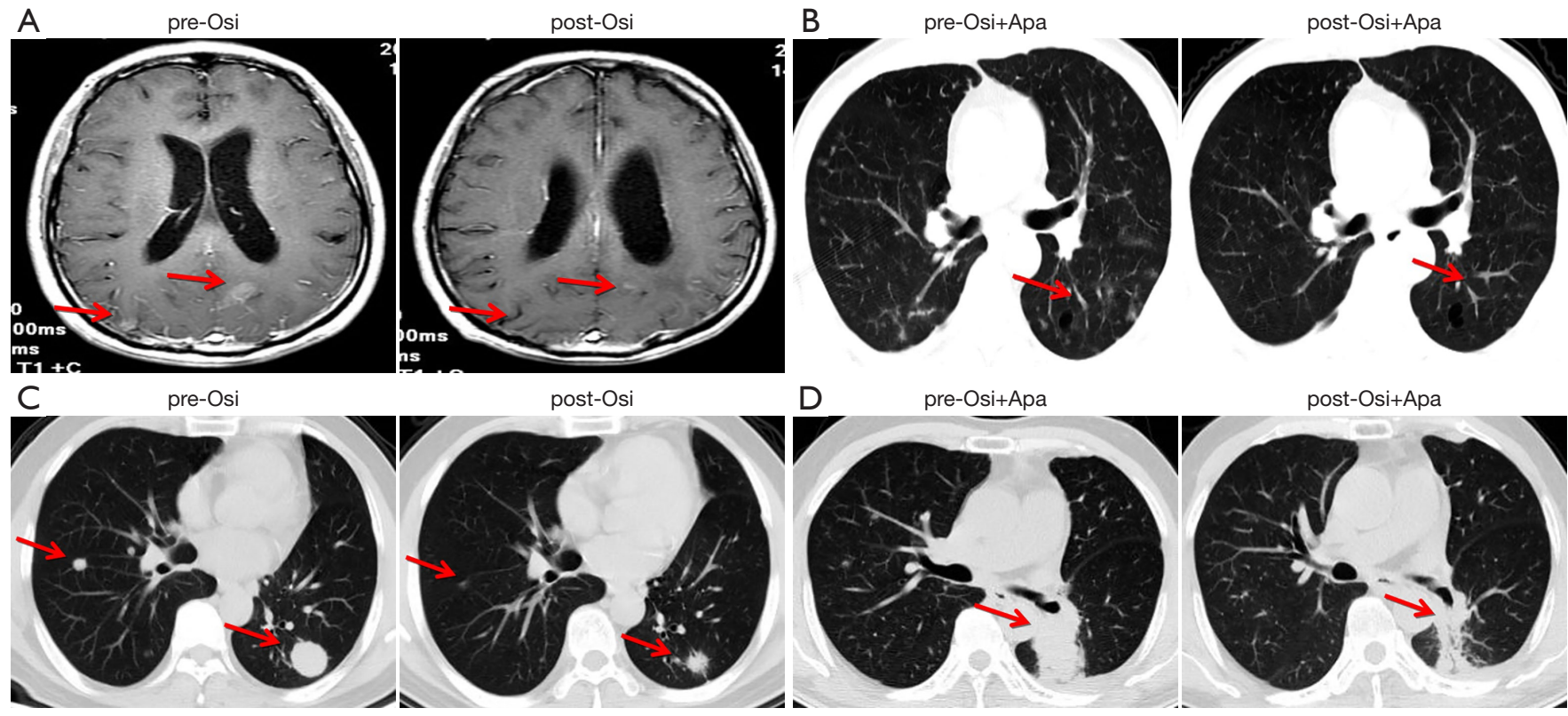

Figure 4 Representative radiological images of two patients treated with osimertinib monotherapy and osimertinib combined with apatinib. (A) Brain MRI before and after osimertinib therapy of patient No. 1 with EGFR T790M, displaying meningeal metastases. The patient had a partial response to osimertinib. (B) After 5 months, the patient acquired resistance to osimertinib, and metastases progressed bilaterally in the lungs. A lung CT scan showed lung metastases had a partial response to the addition of apatinib to osimertinib therapy (PFS $=5$ months). (C) Lung CT scan before and after osimertinib therapy of patient No. 2 with EGFR T790M, displaying a nodule in the left lower lobe. The patient had a partial response to osimertinib. (D) After 5 months, the patient acquired resistance to osimertinib, and the nodule progressed to a primary lung tumor. A lung CT scan showed that the lung nodule had a partial response to the addition of apatinib to osimertinib therapy (PFS $=5$ months). Red arrows indicate changes in lesions before and after treatment.

osimertinib with combined osimertinib and apatinib therapy is worthy of further exploration. We strongly recommend that future larger clinical trials in this population be performed.

The EGFR T790M mutation refers to the mutation of threonine ( $\mathrm{T})$ to methionine $(\mathrm{G})$ at amino acid position 790 in exon 20 of EGFR. Because methionine occupies more space than threonine, this results in the formation of steric hindrance, changing the affinity of ATP to the EGFR kinase domain. As a result, EGFR-TKI-like small molecule drugs can no longer effectively block EGFR activation signals, and they therefore lose their ability to kill tumor cells $(17,18)$. Osimertinib has a 2 -aminopyrimidine structure as a backbone, and binds irreversibly to the C797 amino acid of the EGFR ATP binding site, selected by unsaturated acryloyl chains. It thereby inhibits EGFRsensitive mutations in T790M-mutant tumor cells (16). Our study indicates that the inhibitory rate of PC9 cells was higher than that of $\mathrm{H} 1975$ cells at the same osimertinib concentration, which is consistent with the results of clinical trials. Patients with an EGFR sensitizing mutation who received osimertinib therapy had a significantly longer PFS than patients with acquired T790M mutation-positive NSCLC who received osimertinib therapy $(6,14,17,18)$. Results from one cohort of the AURA I study and the FLAURA phase III trial demonstrated a median PFS of more than 1.5 years for patients with EGFR sensitizing mutation-positive advanced NSCLC who received osimertinib as the first-line treatment $(16,19)$. However, a median PFS of only 10.2 months was seen in the AURA3 phase III trial, amongst patients who acquired EGFR T790M mutation-positive advanced NSCLC after developing secondary resistance to prior first- or secondgeneration EGFR-TKI treatment (6).

The possible mechanisms of secondary resistance to osimertinib include a secondary C797S mutation, loss of T790M, and bypass activation, such as the amplification of MET and HER2 (20). In clinical practice, there is currently no standard therapeutic drug for those patients who both fail multi-line chemotherapy and acquire resistance to the 
Table 2 List of preclinical trials using dual inhibition of the VEGF/VEGFR and EGFR pathways: A+T therapy mode

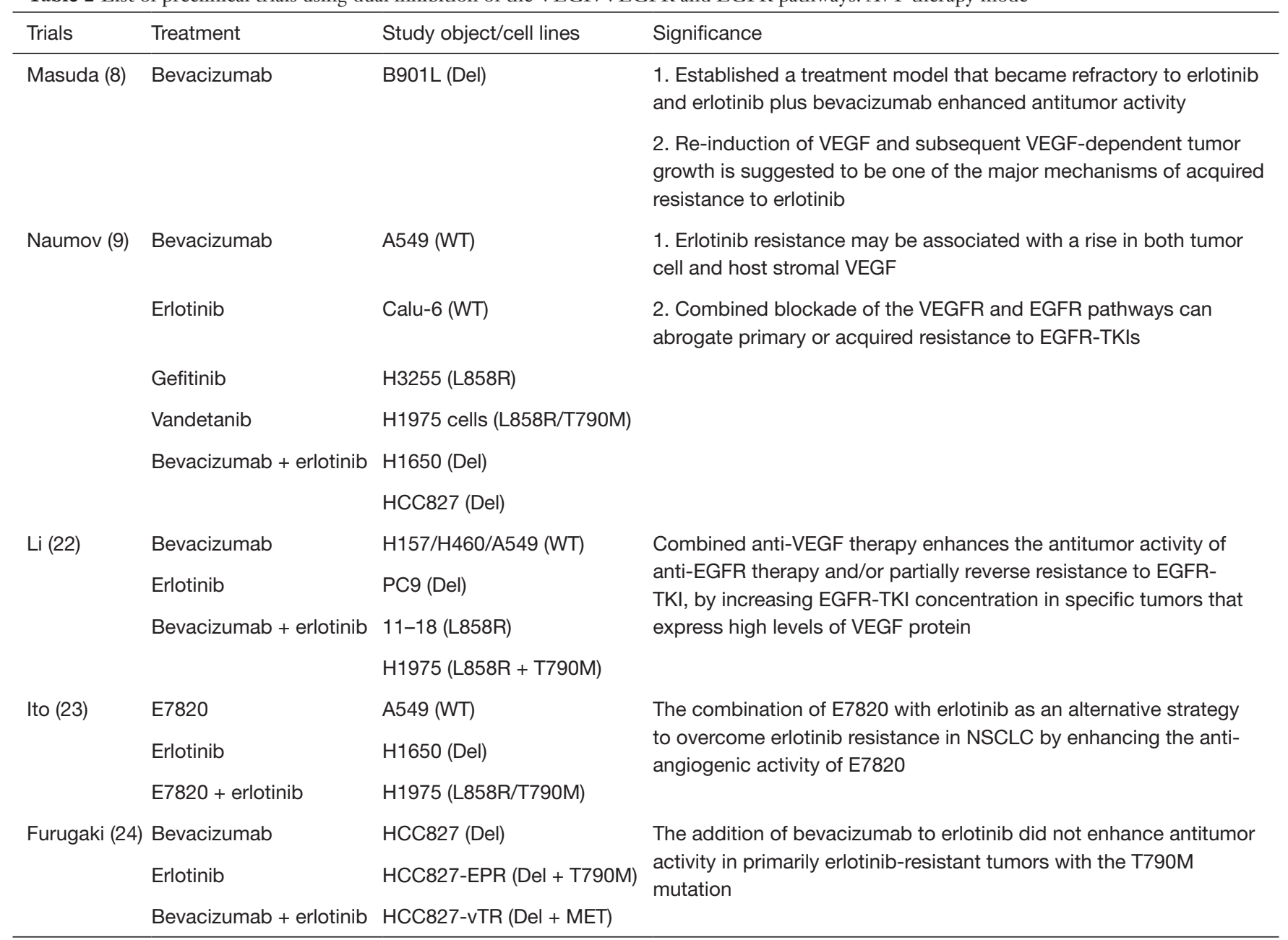

VEGF, vascular endothelial growth factor; EGFR, epidermal growth factor receptor; TKIs, tyrosine kinase inhibitors; WT, EGFR wild-type; Del, EGFR exon 19 deletion; NSCLC, non-small-cell lung cancer; E7820, an angiogenesis inhibitor that decreases integrin- $\alpha 2$ expression.

third-generation EGFR-TKI osimertinib (21). In fact, these patients are encouraged to undergo re-biopsy and gene detection, and clinical trials are strongly recommended. Therefore, it is a considerable challenge to delay the occurrence of resistance to osimertinib, or even restore the sensitivity to osimertinib, especially for patients who have already received multiple chemotherapy regimens.

Before osimertinib was approved for clinical use, many experts explored whether the "A+T" (EGFR-TKIs in combination with anti-angiogenetic drugs) therapy mode could enhance anti-tumor activity, or even reverse resistance to first-generation EGFR-TKIs. The preclinical and clinical trials regarding the "A+T" therapy mode are summarized in Tables 2 and 3, respectively. Most preclinical studies show that combined VEGFR/EGFR pathway blockade can abrogate primary or acquired resistance to EGFR inhibitors $(8,9,22,23)$. Of course, not all preclinical studies support the effectiveness of EGFR-TKIs combined with anti-angiogenic drugs against EGFR-TKI-resistant NSCLC cells. Furugaki et al. indicated that the addition of bevacizumab to erlotinib was ineffective against NSCLC cells or xenograft models that were primarily resistant to erlotinib; however, the combination treatment enhanced the inhibitory effect on T790M mutation- or MET amplification- positive NSCLC cells harboring an EGFR sensitizing mutation, as long as their growth remained significantly suppressed by erlotinib (24). Two randomized control studies reported that erlotinib combined with bevacizumab significantly prolonged the median PFS in patients with EGFR sensitizing mutation-positive NSCLC, compared to 
Table 3 List of clinical trials investigating the dual inhibition of the VEGF/VEGFR and EGFR pathways in patients with NSCLC

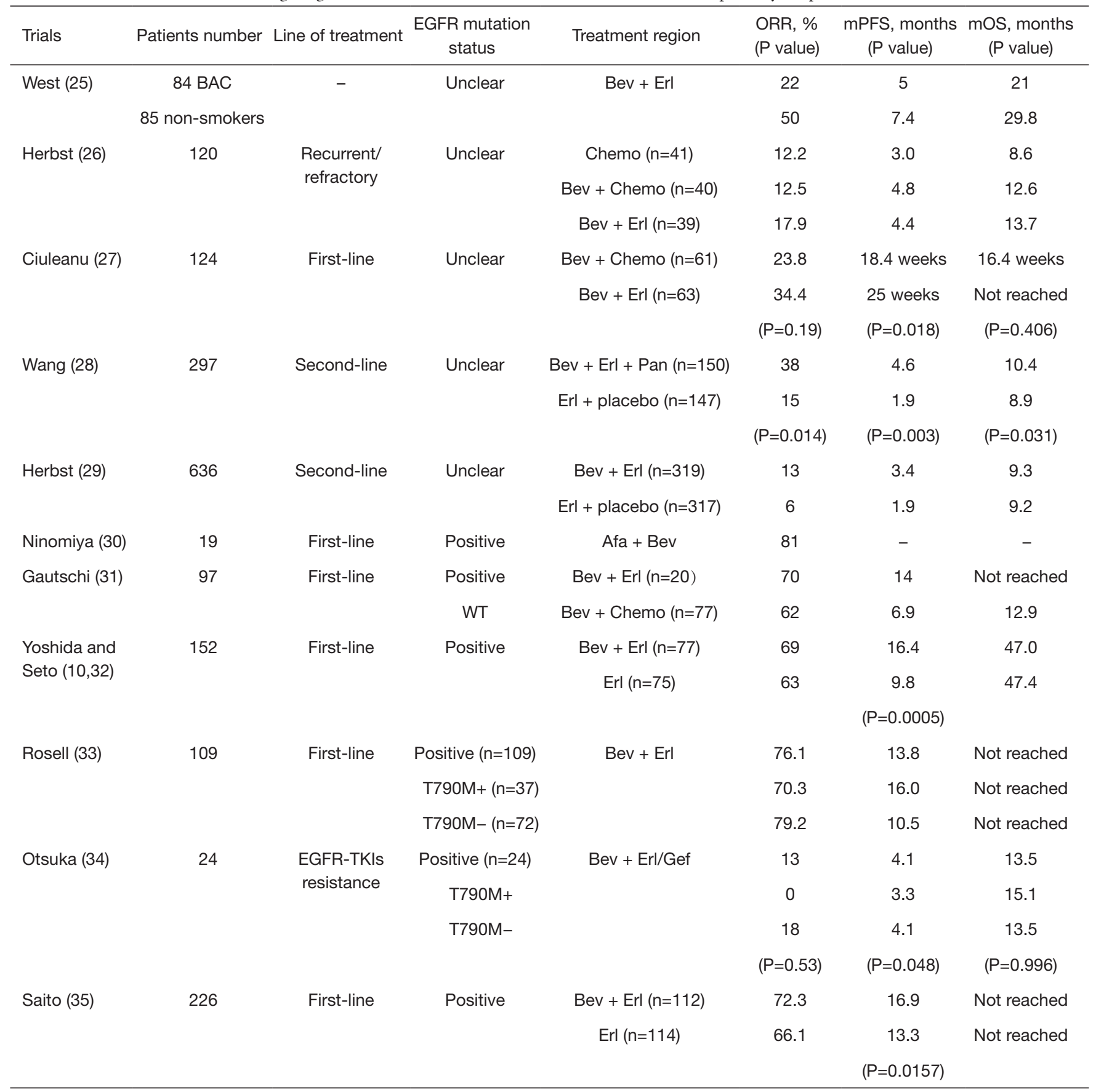

BAC, bronchioloalveolar carcinoma; Erl, erlotinib; Bev, bevacizumab; Chemo, chemotherapy; Pan, panitumumab; Afa, afatinib; Gef, gefitinib; WT, wild-type; bevacizumab in these studies was administered at a dose of $15 \mathrm{mg} / \mathrm{kg}$; erlotinib in these studies was administered at a dose of $150 \mathrm{mg} / \mathrm{day}$; afatinib in these studies was administered at a dose of 40 or $30 \mathrm{mg} /$ day. 
erlotinib alone (16.0-16.9 vs. 9.7-13.3 months) (32,35). We also expect that the results of the ongoing BEVERLY clinical trials will further inform us as to the combined efficacy of bevacizumab and erlotinib (36). Interestingly, a subgroup analysis in the one-armed BELIEF study found that the de novo T790M-positive subgroup had a significantly prolonged median PFS compared to the T790M-negative subgroup (16.0 vs. 10.5 months) when patients were treated with first-line erlotinib combined to bevacizumab (33). The reason for this observed effect remains unclear.

Based on these aforementioned preclinical and clinical trials, it is clear that EGFR-TKIs combined with antiangiogenic targeted drugs results in superior treatment outcomes compared to EGFR-TKIs alone. Our findings indicate that the anti-tumor efficacy on T790M positive lung adenocarcinoma of osimertinib combined with apatinib is significantly higher than that of osimertinib alone, in both in vitro and in vivo experiments. Apatinib is a potent small molecule VEGFR2 TKI used in second- and third-line treatment of patients with advanced NSCLC, that failed to respond to prior chemotherapy or EGFRTKIs. Several clinical studies have shown that the ORR and disease control rate (DCR) of single drug apatinib, in the second-line of treatment or greater, in advanced NSCLC patients ranged from $8 \%$ to $26 \%$ and from $61.9 \%$ to $95.5 \%$, respectively. The median PFS and OS ranged from 3 to 6.77 months and from 6 to 8.2 months, respectively (37-42). Regarding apatinib combination therapy, several preclinical and clinical studies demonstrated an enhanced anti-tumor efficacy using a combination of apatinib and docetaxel for NSCLC, compared to drug monotherapy (43). Another preclinical study reported by $\mathrm{Li}$ et al. (44) demonstrated that gefitinib plus apatinib strengthened the anti-tumor effect of gefitinib or apatinib alone in H1975 cells, and that the combined administration delayed tumor growth in a xenograft model, and had a stronger effect on the inhibition of the activation of EGFR and VEGFR2 downstream pathway members, compared to drug monotherapy. These results are in accordance with the results of this study, which showed that apatinib monotherapy had a weak and negligible effect on anti-tumor activity in vitro, yet a strong synergetic anti-tumor effect in PC9 or H1975 cells when combined with osimertinib. These results suggest that osimertinib combined with apatinib treatment dually inhibits the EGFR and VEGFR signaling pathways. Lichtenberger et al. (45) have reported that there exists "crosstalk" between the EGFR and VEGF pathways, both of which have synergistic effects on tumor growth. VEGF is downregulated by EGFR inhibition, with one of the reasons for the development of EGFR-TKI resistance being increased expression of the VEGF protein. In the present study, we observed that combination therapy in vitro significantly increased the inhibition of EGFR downstream signaling pathway members PI3K-AKT and RAS-ERK. The in vivo synergetic inhibitory effect could be implemented by improving the tumor microenvironment, increasing the drug concentration of osimertinib in tumor tissues and enhancing the blockade of EGFR downstream signal transduction. We also found that a low concentration of osimertinib combined with apatinib had an efficacy similar to that of a high concentration of osimertinib. This is an important factor for drugs such as those with doselimited toxicity, as combination therapy might result in a decreased and tolerable toxicity, thereby avoiding the severe adverse effects caused by simply increasing dosage.

Currently, there are a limited number of clinical studies that have investigated restoring EGFR-TKI sensitivity by adding an anti-angiogenic drug in combination with an EGFR-TKI. A study by Otsuka et al. (34) showed that EGFR-TKIs re-challenged with bevacizumab resulted in a modestly prolonged PFS and OS for patients with EGFRmutant NSCLC that had acquired resistance to EGFRTKI. The median PFS and OS for T790M-positive versus T790M-negative NSCLC was 3.3 vs. 4.1 months, and 15.1 vs. 13.5 months, respectively. Therefore, using EGFR-TKIs in combination with anti-angiogenic drugs to overcome or reverse EGFT-TKI resistance seems to be a promising strategy.

In the present study, before patients were enrolled, they had previously received multi-line chemotherapeutic regimens and acquired secondary resistance to both erlotinib and osimertinib, despite displaying initial responses to the two drugs. The patients had a PFS range of 10-39 months with erlotinib and 3-6 months with osimertinib, respectively (Table 1). According to our preclinical study, they received a combination therapy of osimertinib ( $80 \mathrm{mg} /$ day) plus apatinib (250-500 mg/day). All patients responded to the combination therapy with a prolonged PFS ranging from 5 to 7 months, which is even longer than the median PFS obtained when these patients with the T790M mutation initially received osimertinib. Of note, these patients did not stop taking osimertinib after the failure of osimertinib; that is, there was no treatment interval or so-called osimertinib re-challenge. This suggests that the addition of apatinib to osimertinib may not only 
enhance the synergistic anti-tumor effect observed in our preclinical study, but also partially restore a patient's resistance to osimertinib. Of course, different osimertinibresistance mechanisms might impact the efficacy of this combination therapy, therefore large clinical studies based on molecular detection are warranted. The most common grade 3 adverse events were fatigue, oral mucositis, and anorexia, which usually required symptomatic treatment to avoid interruption or discontinuity in the study.

In conclusion, apatinib can significantly increase the anti-tumor effect of osimertinib for EGFR T790M mutation-positive lung adenocarcinoma, by means of the synergic blockade of the activation of ERK and AKT signal transduction downstream of EGFR. The clinical application of osimertinib combined with apatinib as a treatment choice for osimertinib-resistant patients is promising. Our findings provide a strong basis for the design of clinical trials for this purpose.

\section{Acknowledgments}

Funding: This study was supported by grants from the National Natural Science Foundation of China (grant No. 81501990).

\section{Footnote}

Conflicts of Interest: All authors have completed the ICMJE uniform disclosure form (available at http://dx.doi. org/10.21037/tcr.2019.09.35). The authors have no conflicts of interest to declare.

Ethical Statement: The authors are accountable for all aspects of the work in ensuring that questions related to the accuracy or integrity of any part of the work are appropriately investigated and resolved. This study was conducted in accordance with the Declaration of Helsinki (as revised in 2013). This study was approved by the Ethics Committee of Shengjing Hospital of China Medical University (approval ID: 2015PS167K), and all of the patients have signed the informed consent forms.

Open Access Statement: This is an Open Access article distributed in accordance with the Creative Commons Attribution-NonCommercial-NoDerivs 4.0 International License (CC BY-NC-ND 4.0), which permits the noncommercial replication and distribution of the article with the strict proviso that no changes or edits are made and the original work is properly cited (including links to both the formal publication through the relevant DOI and the license). See: https://creativecommons.org/licenses/by-nc-nd/4.0/.

\section{References}

1. Novello S, Barlesi F, Califano R, et al. Metastatic nonsmall-cell lung cancer: ESMO Clinical practice Guidelines for diagnosis, treatment and follow-up. Ann Oncol 2016;27:v1-27.

2. Savas P, Hughes B, Solomon B. Targeted therapy in lung cancer: IPASS and beyond, keeping abreast of the explosion of targeted therapies for lung cancer. J Thorac Dis 2013;5:S579-92.

3. Wu YL, Zhou C, Hu CP, et al. Afatinib versus cisplatin plus gemcitabine for first-line treatment of Asian patients with advanced non-small-cell lung cancer harbouring EGFR mutations (LUX-Lung 6): an open-label, randomised phase 3 trial. Lancet Oncol 2014;15:213-22.

4. Jänne PA, Yang JC, Kim DW, et al. AZD9291 in EGFR inhibitor-resistant non-small-cell lung cancer. $\mathrm{N}$ Engl J Med 2015;372:1689-99.

5. Liu Y, Sun L, Xiong ZC, et al. Meta-analysis of the impact of de novo and acquired EGFR T790M mutations on the prognosis of patients with non-small cell lung cancer receiving EGFR-TKIs. Onco Targets Ther 2017;10:2267-79.

6. Mok TS, Wu YL, Ahn MJ, et al. Osimertinib or PlatinumPemetrexed in EGFR T790M-Positive Lung Cancer. N Engl J Med 2017;376:629-40.

7. Sun L, Ma JT, Zhang SL, et al. Efficacy and safety of chemotherapy or tyrosine kinase inhibitors combined with bevacizumab versus chemotherapy or tyrosine kinase inhibitors alone in the treatment of non-small cell lung cancer: a systematic review and meta-analysis. Med Oncol 2015;32:473.

8. Masuda C, Yanagisawa M, Yorozu K, et al. Bevacizumab counteracts VEGF-dependent resistance to erlotinib in an EGFR-mutated NSCLC xenograft model. Int J Oncol 2017;51:425-34.

9. Naumov GN, Nilsson MB, Cascone T, et al. Combined vascular endothelial growth factor receptor and epidermal growth factor receptor (EGFR) blockade inhibits tumor growth in xenograft models of EGFR inhibitor resistance. Clin Cancer Res 2009;15:3484-94.

10. Yoshida K, Yamada Y. Erlotinib alone or with bevacizumab as first-line therapy in patients with advanced nonsquamous non-small-cell lung cancer harboring EGFR 
mutations (JO25567): an open-label, randomized, multicenter, phase II study. Transl Lung Cancer Res 2015;4:217-9.

11. Dickson PV, Hamner JB, Sims TL, et al. Bevacizumabinduced transient remodeling of the vasculature in neuroblastoma xenografts results in improved delivery and efficacy of systemically administered chemotherapy. Clin Cancer Res 2007;13:3942-50.

12. Scott LJ. Apatinib: A Review in Advanced Gastric Cancer and Other Advanced Cancers. Drugs 2018;78:747-58.

13. Scott AJ, Messersmith WA, Jimeno A. Apatinib: a promising oral antiangiogenic agent in the treatment of multiple solid tumors. Drugs Today (Barc) 2015;51:223-9.

14. Mi YJ, Liang YJ, Huang HB, et al. Apatinib (YN968D1) reverses multidrug resistance by inhibiting the efflux function of multiple ATP-binding cassette transporters. Cancer Res 2010;70:7981-91.

15. Zhang L, Shi M, Huang C, et al. A phase II, multicenter, placebo controlled trial of apatinib in patients with advanced nonsquamous non-small cell lung cancer (NSCLC) after two previous treatment regimens. J Clin Oncol 2012;30:7548.

16. Soria JC, Ohe Y, Vansteenkiste J, et al. Osimertinib in Untreated EGFR-Mutated Advanced Non-Small-Cell Lung Cancer. N Engl J Med 2018;378:113-25.

17. Pao W, Miller VA, Politi KA, et al. Acquired resistance of lung adenocarcinomas to gefitinib or erlotinib is associated with a second mutation in the EGFR kinase domain. PLoS Med 2005;2:e73.

18. Cross DA, Ashton SE, Ghiorghiu S, et al. AZD9291, an irreversible EGFR TKI, overcomes T790M-mediated resistance to EGFR inhibitors in lung cancer. Cancer Discov 2014;4:1046-61.

19. Ramalingam SS, Yang JC, Lee CK, et al. Osimertinib As First-Line Treatment of EGFR Mutation-Positive Advanced Non-Small-Cell Lung Cancer. J Clin Oncol 2018;36:841-9.

20. Nie K, Jiang H, Zhang C, et al. Mutational Profiling of Non-Small-Cell Lung Cancer Resistant to Osimertinib Using Next-Generation Sequencing in Chinese Patients. Biomed Res Int 2018;2018:9010353.

21. Ettinger DS, Aisner DL, Wood DE, et al. NCCN Guidelines Insights: Non-Small Cell Lung Cancer, Version 5.2018. J Natl Compr Canc Netw 2018;16:807-21.

22. Li H, Takayama K, Wang S, et al. Addition of bevacizumab enhances antitumor activity of erlotinib against non-small cell lung cancer xenografts depending on VEGF expression. Cancer Chemother Pharmacol
2014;74:1297-305.

23. Ito K, Semba T, Uenaka T, et al. Enhanced anti-angiogenic effect of E7820 in combination with erlotinib in epidermal growth factor receptor-tyrosine kinase inhibitor-resistant non-small-cell lung cancer xenograft models. Cancer Sci 2014;105:1023-31.

24. Furugaki K, Fukumura J, Iwai T, et al. Impact of bevacizumab in combination with erlotinib on EGFRmutated non-small cell lung cancer xenograft models with T790M mutation or MET amplification. Int J Cancer 2016;138:1024-32.

25. West HL, Moon J, Wozniak AJ, et al. Paired Phase II Studies of erlotinib/bevacizumab for advanced bronchioloalveolar carcinoma or never smokers with advanced non-small-cell lung cancer: SWOG S0635 and S0636 Trials. Clin Lung Cancer 2018;19:84-92.

26. Herbst RS, O'Neill VJ, Fehrenbacher L, et al. Phase II study of efficacy and safety of bevacizumab in combination with chemotherapy or erlotinib compared with chemotherapy alone for treatment of recurrent or refractory non-small-cell lung cancer. J Clin Oncol 2007;25:4743-50.

27. Ciuleanu T, Tsai CM, Tsao CJ, et al. A phase II study of erlotinib in combination with bevacizumab versus chemotherapy plus bevacizumab in the first-line treatment of advanced non-squamous non-small cell lung cancer. Lung Cancer 2013;82:276-81.

28. Wang $\mathrm{Y}$, Wang $\mathrm{H}$, Jiang $\mathrm{Y}$, et al. A randomized phase III study of combining erlotinib with bevacizumab and panitumumab versus erlotinib alone as second-line therapy for Chinese patients with non-small-cell lung cancer. Biomed Pharmacother 2017;89:875-9.

29. Herbst RS, Ansari R, Bustin F, et al. Efficacy of bevacizumab plus erlotinib versus erlotinib alone in advanced nonsmall-cell lung cancer after failure of standard first-line chemotherapy (BeTa): a double-blind, placebo controlled, phase 3 trial. Lancet 2011;377:1846-54.

30. Ninomiya T, Nogami N, Kozuki T, et al. A phase I trial of afatinib and bevacizumab in chemo-naïve patients with advanced non-small-cell lung cancer harboring EGFR mutations: Okayama Lung Cancer Study Group Trial 1404. Lung Cancer 2018;115:103-8.

31. Gautschi O, Mach N, Rothschild SI, et al. Bevacizumab, pemetrexed, and cisplatin, or bevacizumab and erlotinib for patients with advanced non-small-cell lung cancer stratified by epidermal growth factor receptor mutation: Phase II Trial SAKK19/09. Clin Lung Cancer 2015;16:358-65. 
32. Seto T, Kato T, Nishio M, et al. Erlotinib alone or with bevacizumab as first-line therapy in patients with advanced non-squamous non-small-cell lung cancer harbouring EGFR mutations (JO25567): an open-label, randomised, multicentre, phase 2 study. Lancet Oncol 2014;15:1236-44.

33. Rosell R, Dafni U, Felip E, et al. Erlotinib and bevacizumab in patients with advanced non-small-cell lung cancer and activating EGFR mutations (BELIEF): an international, multicentre, single-arm, phase 2 trial. Lancet Respir Med 2017;5:435-44.

34. Otsuka K, Hata A, Takeshita J, et al. EGFR-TKI rechallenge with bevacizumab in EGFR-mutant nonsmall cell lung cancer. Cancer Chemother Pharmacol 2015;76:835-41.

35. Saito H, Fukuhara T, Furuya N, et al. Erlotinib plus bevacizumab versus erlotinib alone in patients with EGFR-positive advanced non-squamous non-small-cell lung cancer (NEJ026): interim analysis of an open-label, randomised, multicentre, phase 3 trial. Lancet Oncol 2019;20:625-35

36. Gridelli C, Rossi A, Ciardiello F, et al. BEVERLY: Rationale and design of a randomized open-label phase III trial comparing bevacizumab plus erlotinib versus erlotinib alone as first-line treatment of patients with EGFRmutated advanced nonsquamous non-small-cell lung cancer. Clin Lung Cancer 2016;17:461-5.

37. Wu D, Liang L, Nie L, et al. Efficacy, safety and predictive indicators of apatinib after multilines treatment in advanced nonsquamous nonsmall cell lung cancer:

Cite this article as: Liu Y, Xiong ZC, Sun X, Sun L, Zhang SL, Ma JT, Han CB. Impact of apatinib in combination with osimertinib on EGFR T790M-positive lung adenocarcinoma. Transl Cancer Res 2019;8(5):2151-2163. doi: 10.21037/tcr.2019.09.35
Apatinib treatment in nonsquamous NSCLC. Asia Pac J Clin Oncol 2018;14:446-52.

38. Fang S, Zhang M, Wei G, et al. Apatinib as a third- or further- line treatment in patients with advanced NSCLC harboring wild-type EGFR. Oncotarget 2017;9:7175-81.

39. Yang C, Feng W, Wu D. Apatinib for advanced nonsmallcell lung cancer: A retrospective case series analysis. J Cancer Res Ther 2018;14:159-62.

40. Xu J, Liu X, Yang S, et al. Clinical response to apatinib monotherapy in advanced non-small cell lung cancer. Asia Pac J Clin Oncol 2018;14:264-9.

41. Zeng DX, Wang CG, Lei $W$ et al. Efficiency of low dosage apatinib in post-first-line treatment of advanced lung adenocarcinoma. Oncotarget 2017;8:66248-53.

42. Song Z, Yu X, Lou G, et al. Salvage treatment with apatinib for advanced non-small-cell lung cancer. Onco Targets Ther 2017;10:1821-5.

43. Feng S, Zhang J, Wang Y, et al. Application of liquid chromatography-tandem mass spectrometry to study the effect of docetaxel on pharmacokinetics and tissue distribution of apatinib in mice. J Chromatogr B Analyt Technol Biomed Life Sci 2018;1083:198-203.

44. Li F, Zhu T, Cao B, et al. Apatinib enhances antitumour activity of EGFR-TKIs in non-small cell lung cancer with EGFR-TKI resistance. Eur J Cancer 2017;84:184-92.

45. Lichtenberger BM, Tan PK, Niederleithner H, et al. Autocrine VEGF signaling synergizes with EGFR in tumor cells to promote epithelial cancer development. Cell 2010;140:268-79. 